\title{
Influence of the Geometric Parameters on the Densification Onset Strain of Double-Walled Honeycomb Aluminum under Out-of-Plane Compression
}

\author{
Jian Wang $(i D)$ and Ying Cao \\ College of Locomotive and Rolling Stock Engineering, Dalian Jiaotong University, Dalian 116028, China \\ Correspondence should be addressed to Jian Wang; wangjian_126@163.com
}

Received 2 February 2020; Revised 19 February 2020; Accepted 21 February 2020; Published 18 March 2020

Guest Editor: Shun-Peng Zhu

Copyright (C) 2020 Jian Wang and Ying Cao. This is an open access article distributed under the Creative Commons Attribution License, which permits unrestricted use, distribution, and reproduction in any medium, provided the original work is properly cited.

\begin{abstract}
As a material widely used in various lightweight structures and energy absorbing devices, honeycomb aluminum has high specific stiffness and specific strength, excellent energy absorption capacity, and vibration damping. When evaluating the energy absorption of honeycomb aluminum under out-of-plane compression, platform stress and onset strain of densification have become important parameters studied by many scholars. In this work, based on the theory that the energy absorption efficiency determines the densification onset strain, the influence of the geometric design parameters of honeycomb aluminum on the onset strain of out-of-plane quasi-static compression densification is studied. Based on the results of the finite element analysis, the relationship between the onset strain and the geometric design parameters including cell size length and wall thickness is fitted by the least squares method. A linear relationship that the onset strain of densification will decrease with the increase of the reciprocal of cell side length and the onset strain of densification will decrease with the increase of the wall thickness is exhibited in the conclusion. This work can provide a theoretical basis for the calculation of the platform stress in the plastic deformation stage.
\end{abstract}

\section{Introduction}

Honeycomb material is a kind of special porous solid, which contains a large number of cavities and has a regular twodimensional porous structure. Therefore, it has the advantages of low relative density, high specific strength and specific stiffness, good vibration absorption and energy absorption, and sound insulation. As a new lightweight material, which integrates physical function and structural function, honeycomb material is widely used in national defense, shipbuilding, aerospace, construction, transportation, and other fields [1-7]. In recent years, honeycomb aluminum has been used in the anticlimbing device of highspeed EMU to improve the passive safety protection function of rail vehicles because of its buffering and energy absorption characteristics.

The in-plane and out-of-plane mechanical properties of honeycomb materials are mainly concentrated in the elastic deformation section and plastic deformation section $[8,9]$, while the research on the mechanical properties of the honeycomb aluminum densification stage is relatively less. Paul and Ramamurty [10] proposed a method for determining the densification onset strain from the intersection of the stress plateau region and the tangent line of the densification zone; Vural and Ravichandran [11] proposed a method to determine the onset strain of densification with the final local minimum strain before the sharp rise of stress. Chan and Xie [12] proposed a method in which the strain at the tangent slope of the densification region is equal to the slope of the elastic region as the onset strain of densification. With the calibrated numerical model, Wang and Liu [13] carried out extensive comparisons between mechanical performances considering the deformation modes, nominal stress, and energy absorption property in terms of honeycomb cells filled with circular aluminum tubes, conventional honeycomb, and multitubes. Zhu et al. [14] proposed a new strain energy critical plane model describing mean stress effects for life prediction under tension-compression, pure 
torsion, and tension-torsion loadings through characterizing the interaction of normal and shear stress-strain behavior on material planes of TC4 alloys. Moreover, more accurate life predictions are presented based on the new model. Xu et al. [15] built a finite element model of aluminum honeycomb by the shell element to simulate the out-of-plane crushing behavior with a constant impact velocity to compare the trigger force, the platform force, the energy absorption, and the compaction strain of the experimental data and make a further study on the material performance. Wang and Lu [16] carried out some quasi-static and dynamic out-of-plane compression experiments for aluminum honeycombs with different geometric configurations to assess their energy absorption properties. The relations between properties and the ratio of span to thickness on plateau stress, specific load, mass specific energy absorption, and volume specific energy absorption were analyzed, which indirectly reflected the relation between energy absorption property and cell density, as well as apparent density.

It is important to study the onset strain of densification to determine the plastic platform stress, and it is also an important parameter to evaluate the energy absorption performance of honeycomb materials. Understanding the key parameters dominating the onset strain of densification is undoubtedly important for practical designs of this kind of materials. In this paper, a finite element model of doublewalled thick honeycomb aluminum is established to study the influence of geometric design parameters including cell size length and wall thickness on the onset strain of densification, and the calculation formula between the onset strain of densification and geometric design parameters are derived by the least squares method.

\section{Determination of Onset Strain of Densification}

The quasi-static compression behavior of honeycomb aluminum can be divided into three parts: elastic deformation section, plastic platform section, and densification section, as shown in Figure 1. When the compression appears in the elastic deformation section, the stress increases linearly with the increase of strain; at the later stage of the elastic deformation, due to the elastic buckling of the honeycomb wall, the stress-strain curve changes nonlinearly until the stress peak, which is called the initial peak stress; the corresponding strain is called the initial strain, which indicates the end of the quasi-static compression elastic stage of the honeycomb aluminum and the beginning of the plastic failure stage.

After the stress reaches the peak point, the stress drops rapidly with the increase of strain. After decreasing to a certain extent, the stress value remains within a stable range. The stress level value of the stable range is called the dynamic peak stress, which indicates the honeycomb aluminum is quasi-statically compressed into the plastic platform section.

When the honeycomb aluminum cell walls begin to contact each other and the matrix material begins to withstand the compressive load, the stress increases sharply. At this moment, the mechanical properties of the honeycomb

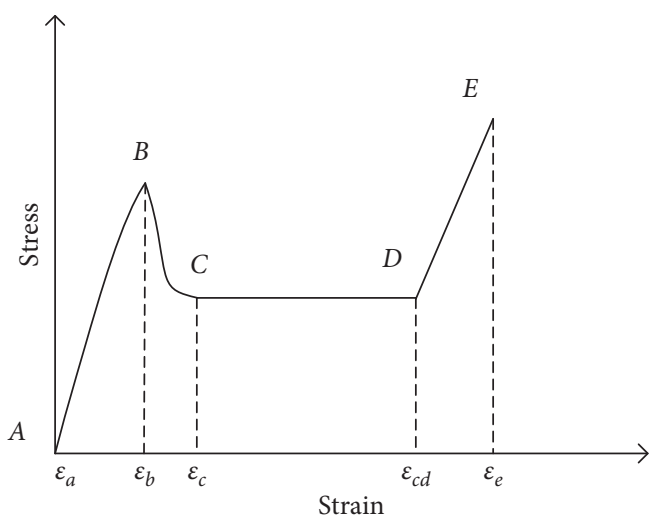

FIgURE 1: Honeycomb aluminum quasi-static compression behavior (AB: elastic section; BD: plastic section; DE: densification section).

material tend to the mechanical properties of the matrix material, which represents the compression behavior from the plastic platform section to the densification section.

Li et al. [17] proposed a concept of the onset strain of densification and defined the turning point of the transition of the honeycomb aluminum plastic platform section to the densification section as the onset strain of densification.

The area enclosed under the curve in the stress-strain diagram shown in Figure 1 is the energy absorption per unit volume of the honeycomb aluminum. The energy absorption efficiency curve of honeycomb aluminum can be plotted in Figure 2. It can be seen from the figure that the energy absorption is mainly concentrated in the platform area before densification. After the compression behavior enters the densification section, the stress value increases sharply and the energy absorption efficiency begins to slow down.

As shown in Figure 2, the energy absorption efficiency increases firstly with the increasing of strain, which corresponds to the elastic deformation section and the plastic platform section of the stress-strain curve. When the curve reaches the peak, the energy absorption efficiency falls rapidly, and the corresponding process is the compression section in which the honeycomb aluminum is gradually compacted.

Based on the method of energy absorption efficiency, the strain energy of any strain point during honeycomb aluminum compression can be written as follows:

$$
E_{c}=\int_{\varepsilon_{0}}^{\varepsilon_{R}} \sigma(\varepsilon) \mathrm{d} \varepsilon
$$

where $\sigma(\varepsilon)$ is the stress at strain of $\varepsilon, \varepsilon_{0}$ is the strain of initial point, and $\varepsilon_{R}$ is an any point on the curve.

The energy absorption efficiency can be defined as the ratio between the strain energy absorbed at any strain point and the stress corresponding to the strain point:

$$
\eta\left(\varepsilon_{R}\right)=\frac{E_{c}}{\left.\sigma_{c}(\varepsilon)\right|_{\varepsilon=\varepsilon_{R}}}=\frac{\int_{\varepsilon_{0}}^{\varepsilon_{R}} \sigma(\varepsilon) \mathrm{d} \varepsilon}{\left.\sigma_{c}(\varepsilon)\right|_{\varepsilon=\varepsilon_{R}}},
$$

where $\left.\sigma_{c}(\varepsilon)\right|_{\varepsilon=\varepsilon_{R}}$ is the stress value corresponding to any strain point. 


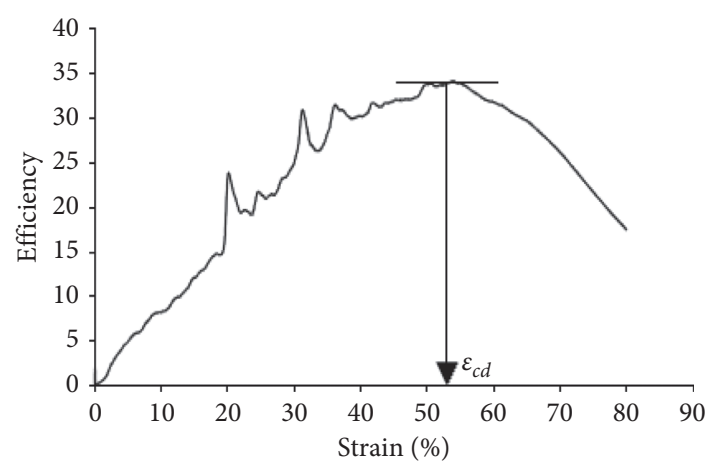

Figure 2: Energy absorption efficiency curve.

Based on the energy absorption efficiency curve of Figure 2, the onset strain of densification is defined as the strain corresponding to the point of maximum energy absorption efficiency:

$$
\left.\frac{\mathrm{d}\left(\eta_{\varepsilon}\right)}{\mathrm{d} \varepsilon}\right|_{\varepsilon=\varepsilon_{D}}=0,
$$

where $\varepsilon_{D}$ is the onset strain of densification. Therefore, the dynamic average stress of the plastic platform section can be obtained by the following formula:

$$
\sigma_{p l}=\frac{\int_{\varepsilon_{0}}^{\varepsilon_{D}} \sigma(\varepsilon) \mathrm{d} \varepsilon}{\varepsilon_{D}-\varepsilon_{0}} .
$$

\section{The Finite Element Model of Double-Walled Thick Honeycomb Aluminum}

The general commercial honeycomb aluminum is a doublewalled thick honeycomb aluminum structure, as shown in Figure 3. Each honeycomb cell has 4 walls with a length of $l$ and a wall thickness of $t$ and 2 walls with a length of $h$ and a wall thickness of $2 t$. The direction perpendicular to the double-thickness of the cell wall is defined as the $\mathrm{W}$ direction, and the direction parallel to the double-thickness of the cell wall is defined as the $L$ direction, which are collectively referred to as the coplanar direction, and the direction parallel to the depth of the cell wall is referred to as $T$ direction, the out-of-plane direction. The so-called out-ofplane compression is to apply a compressive force in this direction.

A finite element model of quasi-static compression of double-walled thick honeycomb aluminum is built, as shown in Figure 4. Four-node shell element is used in the honeycomb aluminum model, and the rigid wall is eightnode solid element.

The $x$ - and $y$-axis directions correspond to the $W$ and $L$ directions of the honeycomb aluminum, respectively, and the $z$-axis direction corresponds to the out-of-plane direction, $T$ direction of the honeycomb aluminum. The honeycomb aluminum cell structure is simplified to a regular hexagon, the cell side length $h=l=6 \mathrm{~mm}$, the expansion angle $\theta=30^{\circ}$, the cell side $l$ thickness is $t=0.32 \mathrm{~mm}$, the $h$ side thickness is $t=0.64 \mathrm{~mm}$, and the cell wall depth is

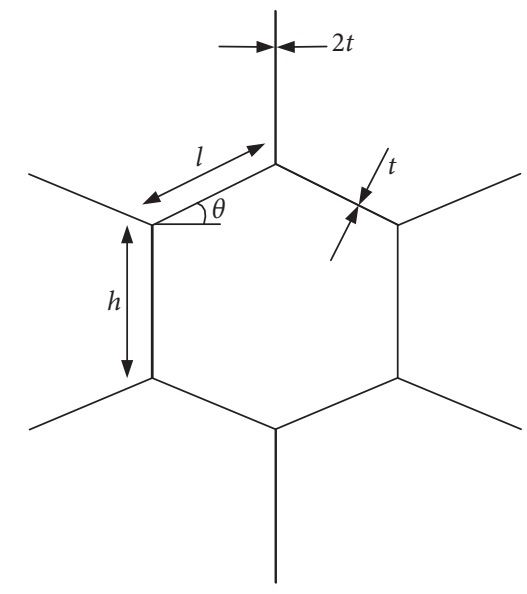

Figure 3: Hexagon double-walled honeycomb structure.

$L_{D}=60 \mathrm{~mm}$. The material properties of the honeycomb aluminum matrix material and the rigid wall material in the finite element model are shown in Table 1.

Using PAM-CRASH to analyze the model, set the constraints, loads, and contact parameters in the finite element model of the honeycomb aluminum. Constrain all the degrees of freedom of the fixed rigid wall, ensuring that the fixed rigid wall does not move and deform during the compression process. Constrain the degrees of freedom of the moving rigid wall in $X$ and $Y$ direction, and the initial velocity of the moving rigid wall is set to $v=10 \mathrm{~m} / \mathrm{s}$ for free fall motion along the $Z$ direction. The contact type between the honeycomb aluminum and the rigid wall is set as the master-slave contact, the contact thickness is set to half of the single wall thickness $0.16 \mathrm{~mm}$, the contact type between the honeycomb aluminum itself is set to self-contact, and the contact thickness is set to the single wall thickness value $0.32 \mathrm{~mm}$.

\section{Data Analysis}

The finite element model is imported into PAM-CRASH environment to be calculated. Some data such as the macrocompression length, the reaction force, and the internal energy absorption of the honeycomb aluminum finite element model are extracted and analyzed.

The stress value at any point on the compressed honeycomb aluminum generally is

$$
\sigma=\frac{F}{A}
$$

where $A$ is the area of out-of-plane compression contact surface and $F$ is the reaction force extracted from analysis results.

The strain value at any point on the compressed honeycomb aluminum can be defined as follows:

$$
\varepsilon=\frac{l_{D}}{L_{D}},
$$

where $l_{D}$ is the compressed length of the honeycomb aluminum and $L_{D}$ is the length in the out-of-plane direction of the honeycomb aluminum. 

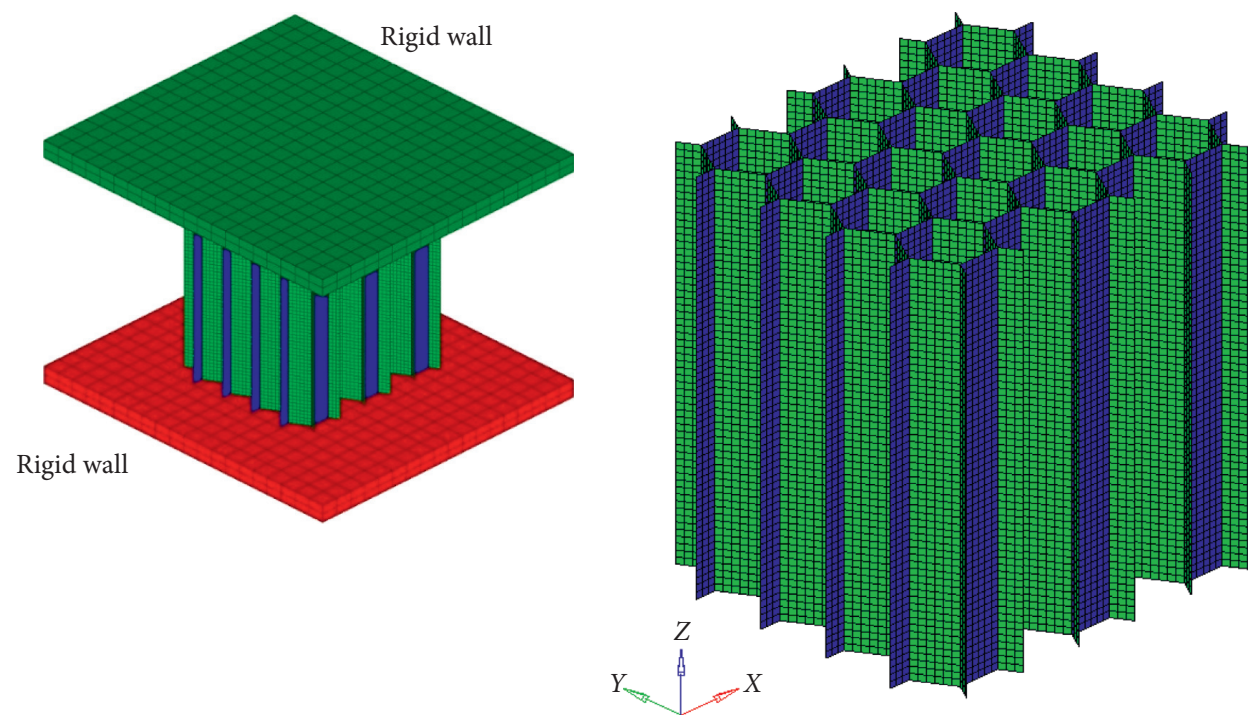

(a)

(b)

Figure 4: The finite element model of honeycomb aluminum. (a). Overall model. (b). Honeycomb aluminum.

TABLe 1: Material properties parameters.

\begin{tabular}{lcccc}
\hline Item & Density $\rho_{s}\left(\mathrm{~kg} / \mathrm{m}^{3}\right)$ & Elastic modulus $E_{s}(\mathrm{MPa})$ & Poisson's ratio $\gamma$ & Yield stress $\sigma_{y s}(\mathrm{MPa})$ \\
\hline Honeycomb aluminum & 2700 & 71000 & 0.33 & 80 \\
Rigid wall & 7800 & 206000 & 0.3 & 245 \\
\hline
\end{tabular}

Combining equations (5) and (6), it can be used to derive the formula for calculating the internal energy absorption of honeycomb aluminum compression:

$$
I=\int_{l_{0}}^{l_{R}} F\left(l_{D}\right) \mathrm{d} l=A L_{D} \int_{\varepsilon_{0}}^{\varepsilon_{R}} \sigma(\varepsilon) \mathrm{d} \varepsilon,
$$

where $I$ is the variable of internal energy absorption of the model.

The internal energy absorption efficiency can be derived from equations (3)- (8):

$$
\eta\left(\varepsilon_{R}\right)=\frac{\int_{\varepsilon_{0}}^{\varepsilon_{R}} \sigma(\varepsilon) \mathrm{d} \varepsilon}{\left.\sigma(\varepsilon)\right|_{\varepsilon=\varepsilon_{R}}}=\frac{\int_{l_{0}}^{l_{R}} F\left(l_{D}\right) \mathrm{d} l}{\left.L_{D} F\right|_{l_{D}=l_{R}}}=\frac{\left.I\right|_{l_{D}=l_{R}}}{\left.L_{D} F\right|_{l_{D}=l_{R}}} .
$$

Figures 5 and 6 show the stress-strain curve and energy absorption efficiency-strain curve of the honeycomb aluminum in out-of-plane quasi-static compressive load case.

It can be seen from Figure 5 that the out-of-plane quasistatic compression behavior of honeycomb aluminum is consistent with the theoretical description of honeycomb aluminum compression deformation and is clearly divided into elastic deformation section, plastic section, and densified deformation section. Figure 6 shows the energy absorption-strain curve of honeycomb aluminum. The figure shows that the energy absorption efficiency increases with the increase of strain. When the energy absorption efficiency increases to the peak, it declines rapidly. At this point, the strain corresponding to the peak is defined as the onset strain of the densification point.

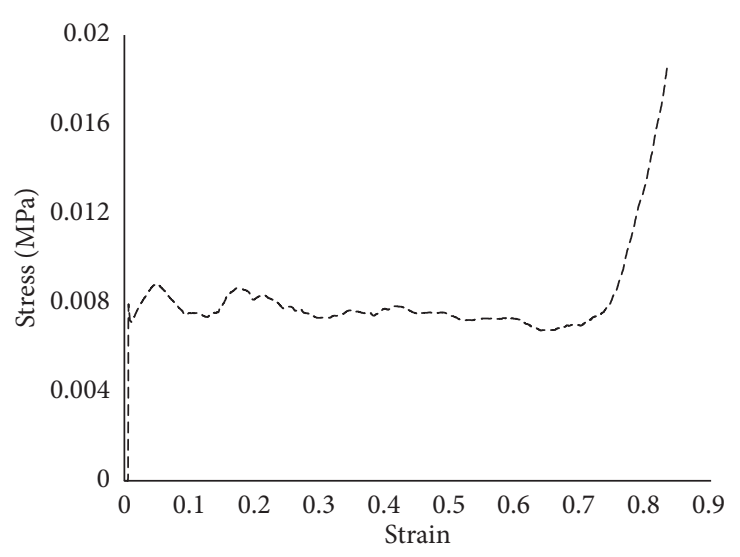

FIGURE 5: Stress-strain curve of honeycomb aluminum in out-ofplane quasi-static compressive load case.

\section{Effect of Dimension Variables on Onset Strain of Densification}

According to the concept of densification strain [18], there is a certain linear relationship between densification strain and cell side length and cell wall thickness. Based on the finite element model of honeycomb aluminum, the influence of cell side length and cell wall thickness on densification onset strain is studied, and the relationship between densification onset strain and cell size is summarized by least square fitting. The formula of onset strain of densification is obtained. 


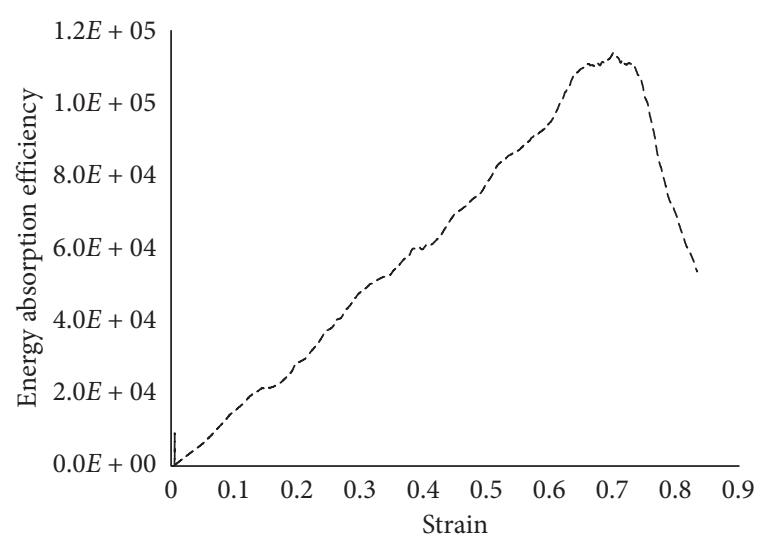

FIGURE 6: Energy absorption efficiency-strain curve of honeycomb aluminum in out-of-plane quasi-static compressive load case.

5.1. Influence of Cell Side Length. As shown in Figure 4, the finite element model of double-walled thickness honeycomb aluminum is established, which keeps the cell shape and wall thickness unchanged, only changes the cell side length, and carries out numerical simulation calculation for the finite element model of different cell side lengths. The stress-strain curves of honeycomb aluminum with different cell side lengths are plotted, as shown in Figure 7, and the corresponding data are shown in Table 2. It can be seen from the figure that with the increase of the cell side length of the honeycomb aluminum, the stress peak value of the elastic deformation section of the honeycomb aluminum increases, the dynamic stress mean value of the plastic collapse section increases, and the strain value of the transition from the plastic deformation section to the densification stage also increases.

In order to facilitate the comparison, the reciprocal of the cell side length is selected as the variable to study the internal relationship between the cell side length and the onset strain of densification. The relationship between the onset strain of densification and the reciprocal of cell side length is shown in Figure 8. It can be seen from the figure that the onset strain of densification decreases with the increases of the reciprocal of cell side length. Therefore, the onset strain of densification increases with the increase of cell side length.

Based on the correlative data of onset strain of densification and cell side length, it is assumed that there is the following relationship between them:

$$
\varepsilon_{D}=b-a \frac{1}{l}
$$

The data of the onset strain of densification and the reciprocal of the cell side length are linearly fitted by the least square method, and the fitting line segments between them are obtained. The fitting parameters are, respectively, $a=1.0804$ and $b=0.8932$. The linear relationship expression between the onset strain of densification and the reciprocal of the cell side length can be obtained as follows:

$$
\varepsilon_{D}=0.8932-1.0804 \frac{1}{l} .
$$

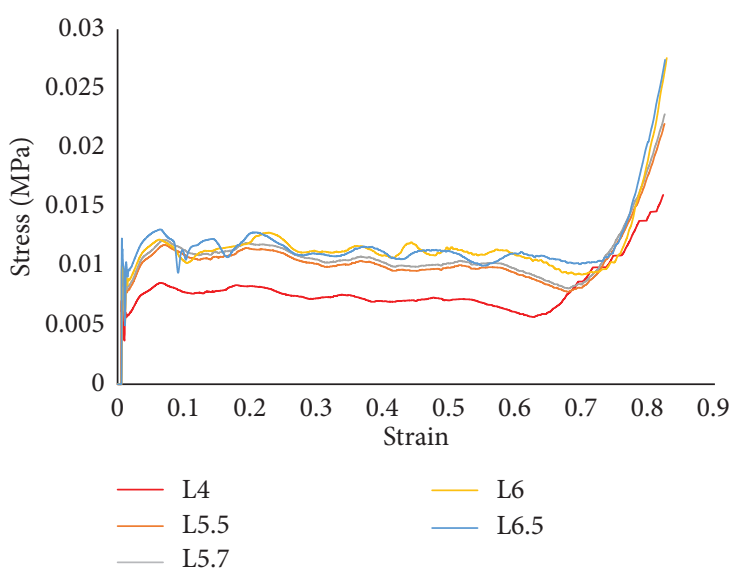

FIgURE 7: Stress-strain curves of honeycomb aluminum with different cell side lengths.

TABle 2: Onset strain of densification under different cell side lengths.

\begin{tabular}{lcc}
\hline $\begin{array}{l}\text { Cell side length } \\
(\mathrm{mm})\end{array}$ & $\begin{array}{c}\text { Reciprocal of } \\
\text { length }\left(\mathrm{mm}^{-1}\right)\end{array}$ & $\begin{array}{c}\text { Onset strain of } \\
\text { densification } \varepsilon_{D}\end{array}$ \\
\hline 4 & 0.250000 & 0.655811 \\
5.5 & 0.181818 & 0.702188 \\
5.7 & 0.175439 & 0.710015 \\
6 & 0.166667 & 0.723189 \\
6.5 & 0.153846 & 0.730632 \\
\hline
\end{tabular}

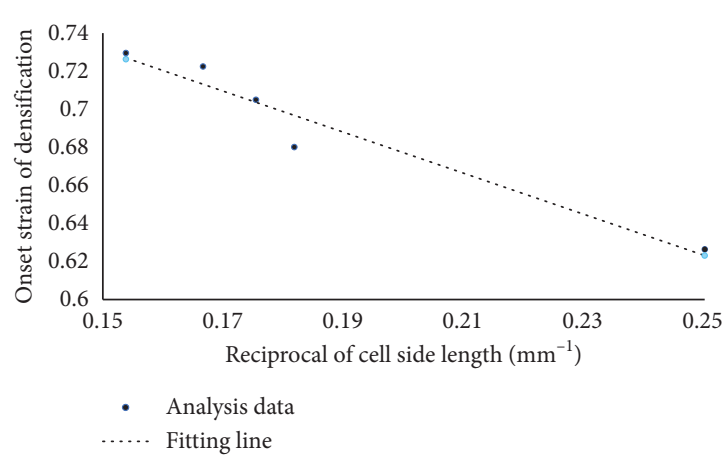

FIGURE 8: Influence curve of cell side length on the onset of densification.

The correlation coefficient in the least squares method is used to represent the approximation degree between data and can be used to measure the dispersion of discrete points relative to standard deviation. In the abovementioned fitting results, the square $R$ of the correlation coefficient between the onset strain of densification and the cell side length data fitting is 0.9448 .

5.2. Influence of Wall Thickness. Using the abovementioned finite element model, set different cell wall thicknesses, evenly arrange the cell wall thickness in the range of $0.4 \sim 0.6$, and set 200 groups of different thickness. A series of stressstrain curves of honeycomb aluminum are calculated, some 


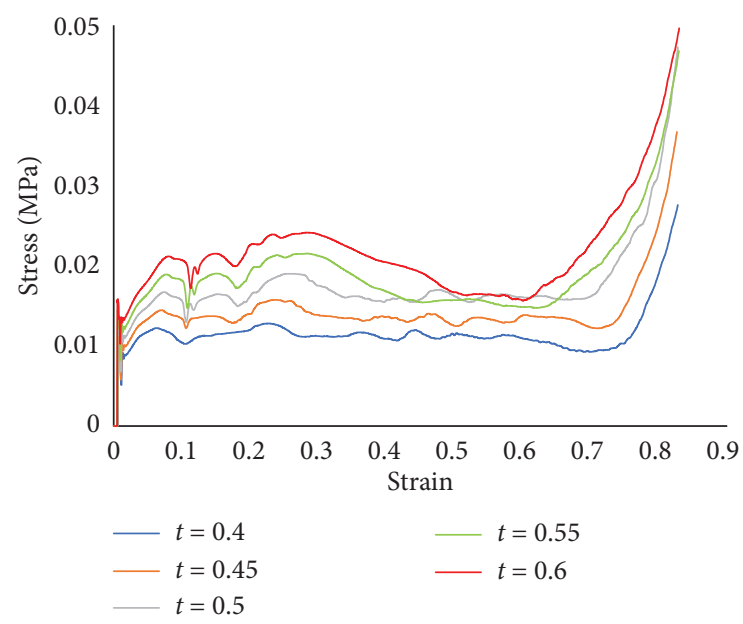

FIgURE 9: Stress-strain curves of honeycomb aluminum under different cell wall thicknesses.

of which are shown in Figure 9 and the corresponding values are shown in Table 3. It can be seen from the calculation results that, with the increase of the thickness of the honeycomb aluminum cell wall, the peak value of the stress in the elastic deformation section of the honeycomb aluminum increases, the mean value of the dynamic stress in the plastic collapse section increases, and the strain value in the transition from the plastic deformation section to the densification stage decreases.

Based on the calculation method of energy absorption efficiency of honeycomb aluminum compression, combined with the internal energy absorption efficiency curve, the densification onset strain corresponding to the wall thickness of each group of honeycomb aluminum cells can be obtained, as shown in Figure 10. It can be seen from the data in the figure that the onset strain of densification decreases with the increase of cell wall thickness. It is assumed that the effect of cell wall thickness on the onset strain of densification is linear, and a linear expression between the two is proposed:

$$
\varepsilon_{D}=1-\alpha t
$$

where $\alpha$ is a fixed coefficient.

The trend of the curve is analyzed, and the relationship between the two is fitted to the linear relationship, as shown in Figure 10, by the least squares method, and a linear expression between the two is given:

$$
\varepsilon_{D}=1-0.645 t
$$

In the data fitting process, the square value of the correlation coefficient between the linear simulation value obtained by the least square fitting and the finite element simulation result is $R^{2}=0.8599$.
TABLE 3: Onset of densification under different thicknesses of wall.

\begin{tabular}{lc}
\hline Wall thickness $(\mathrm{mm})$ & Onset strain of densification $\varepsilon_{D}$ \\
\hline 0.4 & 0.626371 \\
0.45 & 0.703814 \\
0.5 & 0.705058 \\
0.55 & 0.722544 \\
0.6 & 0.729530 \\
\hline
\end{tabular}

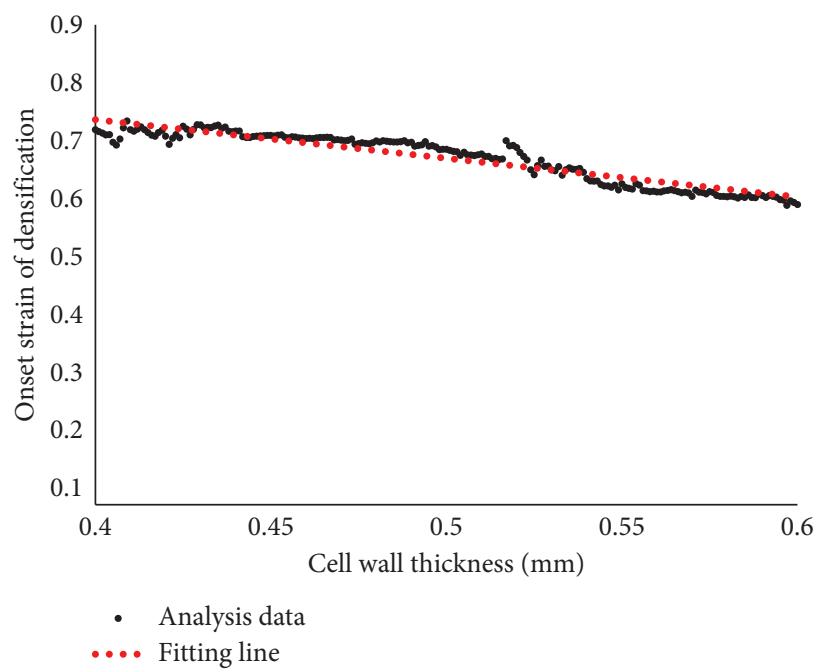

FIGURE 10: Influence of wall thickness on the onset strain of densification.

\section{Conclusion}

In this work, the finite element analysis method is used to study the heterogeneous compression performance of honeycomb aluminum, and the energy absorption efficiency is combined to determine the onset strain of densification. The influence of size length on the onset strain of densification of honeycomb aluminum is discussed. Based on the least squares method, the relationship between the onset strain of densification and the length of the cell side is derived, and the onset strain of densification will decrease with the increase of the reciprocal of the cell side length; the expression of the onset strain of densification and the wall thickness is derived, and the onset strain of densification will decrease with the increase of the wall thickness. The research results enrich the research content of the honeycomb aluminum in out-of-plane compression densification stage and provide a reference for the design of out-ofplane performance of honeycomb aluminum.

\section{Data Availability}

All data generated or analyzed during this study are included in this article. 


\section{Conflicts of Interest}

The authors declare that they have no conflicts of interest.

\section{Acknowledgments}

The project of the research presented in this paper was supported by the National Key R\&D Program of China (2016YFB1200505) and Liaoning Natural Science Foundation Project (20170540129). The authors would like to acknowledge those funding supports sincerely.

\section{References}

[1] A. Nazir, K. M. Abate, A. Kumar, and J.-Y. Jeng, "A state-ofthe-art review on types, design, optimization, and additive manufacturing of cellular structures," International Journal of Advanced Manufacturing Technology, vol. 104, no. 9-12, pp. 3489-3510, 2019.

[2] H. Y. Chang and F. G. Yuan, "Impact damage imaging in a honeycomb composite panel with iw via Riesz transform (Conference Presentation)," in Proceedings of the SPIE Smart Structures and Materials, Nondestructive Evaluation and Health Monitoring, Society of Photo-Optical Instrumentation Engineers (SPIE) Conference Series, Portland, OR, USA, May 2017.

[3] A. Riccio, A. Raimondo, S. Saputo, A. Sellitto, M. Battaglia, and G. Petrone, "A numerical study on the impact behaviour of natural fibres made honeycomb cores," Composite Structures, vol. 202, no. 10, pp. 909-916, 2018.

[4] X. C. Zhang and L. L. Wang, "Simulation of mechanical properties of aluminum honeycomb panels by ANSYS," Machine Design and Manufacturing Engineering, vol. 46, no. 12, pp. 34-36, 2017.

[5] Y. J. Xin, L. W. Zhang, X. M. Liu, S. L. Cheng, and H. . Li, "Impact test on aluminum honeycomb sandwich panels," Journal of Mechanical Strength, vol. 40, no. 4, pp. 802-809, 2018.

[6] D. Q. Sun, W. H. Zhang, and Y. J. Sun, "Elastic moduli and material efficiency of aluminum honeycomb cores," $\mathrm{Me}$ chanics in Engineering, vol. 30, no. 1, pp. 39-44, 2008.

[7] L. L. Hu and T. X. Yu, "Influence of inertia effect on the energy absorption of hexagonal honeycombs," Acta Armamentarii, vol. 30, no. 2, pp. 24-27, 2009.

[8] A. Ajdari, H. Nayeb-Hashemi, P. Canavan, and G. Warner, "Effect of defects on elastic-plastic behavior of cellular materials," Materials Science \& Engineering: A, vol. 487, no. 1-2, pp. 558-567, 2008.

[9] J. Kee Paik, A. K. Thayamballi, and G. Sung Kim, "The strength characteristics of aluminum honeycomb sandwich panels," Thin-Walled Structures, vol. 35, no. 3, pp. 205-231, 1999.

[10] A. Paul and U. Ramamurty, "Strain rate sensitivity of a closedcell aluminum foam," Materials Science \& Engineering A, vol. 281, no. 1-2, pp. 1-7, 2000.

[11] M. Vural and G. Ravichandran, "Microstructural aspects and modeling of failure in naturally occurring porous composites," Mechanics of Materials, vol. 35, no. 3-6, pp. 523-536, 2003.

[12] K. C. Chan and L. S. Xie, "Dependency of densification properties on cell topology of metal foams," Scripta Materialia, vol. 48, no. 8, pp. 1147-1152, 2003.

[13] Z. G. Wang and J. F. Liu, "Numerical and theoretical analysis of honeycomb structure filled with circular aluminum tubes subjected to axial compression," Composites Part B: Engineering, vol. 165, pp. 626-635, 2019.

[14] S.-P. Zhu, Z.-Y. Yu, Q. Liu, and A. Ince, "Strain energy-based multiaxial fatigue life prediction under normal/shear stress interaction," International Journal of Damage Mechanics, vol. 28, no. 5, pp. 708-739, 2019.

[15] T. S. Xu, Y. B. Sun, S. Q. Ma, M. W. Li, and C. Bi, "Simulation and verification of aluminum honeycomb out-of-plane crushing behavior based on pamcrash," Mechanical Research \& Application, vol. 32, no. 3, pp. 66-69, 2019.

[16] Z. G. Wang and Z. J. Lu, "Experimental assessment on energy absorption property of aluminum honeycomb under out-ofplane compression," Journal of Central South University (Science and Technology), vol. 44, no. 3, pp. 1246-1251, 2013.

[17] Q. M. Li, I. Magkiriadis, and J. J. Harrigan, "Compressive strain at the onset of densification of cellular solids," Journal of Cellular Plastics, vol. 42, no. 5, pp. 371-392, 2006.

[18] L. J. Gibson and M. F. Ashby, Cellular Solids: Structure and Properties, Pergamon Press, Oxford, UK, 1988. 